\title{
Un Aleph en la región más transparente del aire
}

\author{
Patricio Santillán-Doherty, ${ }^{*} \bowtie$ Justino Regalado-Pineda
}

*Editor en Jefe de NCT, Director Médico, Instituto Nacional de Enfermedades Respiratorias

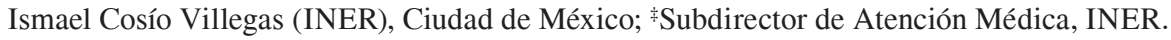
Trabajo recibido: 23-VIII-2016; aceptado: 23-VIII-2016

"El lugar donde están, sin confundirse, todos los lugares del orbe, vistos desde todos los ángulos..." El Aleph. Jorge Luis Borges.

"¿Y qué? -se pregunta Andrei Efimich, abriendo los ojos-. ¿Qué resulta de todo esto? Tenemos los antisépticos, a Koch, a Pasteur, pero en esencia nada ha cambiado en absoluto." La Sala Número Seis. Antón Chéjov.

Cumplir ochenta años no es poca cosa; para las personas significa toda una vida, para una institución médica implica la oportunidad de consolidar su evolución y aquilatar la trascendencia de su quehacer; la oportunidad de convertirse en un verdadero Aleph borgiano de la medicina respiratoria, tal y como lo describe el mismo Jorge Luis en su famoso cuento: «el punto donde convergen todos los puntos».

En la medicina respiratoria nacional todos los puntos convergen en el INER, nuestro Aleph. Celebrar ochenta años de actividad obliga a revisar el camino andado, hacer un recuento de los trabajos hechos y reconocer la evolución lograda para evitar la confusión de la costumbre y la inmovilidad pesimista del personaje chejoviano del Dr. Efimich quien no ve futuro alentador posible en el pabellón seis donde trabaja.

Chéjov supo en vida propia de ese pesimismo por la tuberculosis que padeció, la cual finalmente terminó con su vida de manera prematura. Si bien su novela La Sala Número Seis trata sobre un pabellón dedicado a enfermos mentales, es fácil la analogía con los pabellones antiguos de tuberculosos en donde el aislamiento y el reposo era lo único que se podía ofrecer. La tubercu-

"Este escrito es reproducido con permiso de los autores del libro conmemorativo de los 80 años del Instituto Nacional de Enfermedades Respiratorias Ismael Cosío Villegas". losis, la tisis, el consumo, eran una y la misma. En los siglos XVII-XX la patología tuberculosa era signo chic que corroboraba el carácter melancólico de la época romántica.

Susan Sontag menciona en La Enfermedad y Sus Metáforas cierta similitud entre la tuberculosis y las enfermedades mentales: "Por mucho que se la temiera, la tuberculosis tenía 'pathos'. El tuberculoso, como el enfermo mental de hoy, era la quintaesencia de la vulnerabilidad, un ser poblado de caprichos autodestructivos. Los médicos del siglo XIX y principios del $X X$ se empeñaban en seducir a sus pacientes para que volvieran a la vida. Sus recetas tenían el mismo tenor ilustrado que las que se dan hoy a los enfermos mentales: entorno alegre, aislamiento de toda fuente de estrés y de la familia, régimen sano, ejercicios, reposo".."

Ese es el entorno de pensamiento con el que nació el Sanatorio para Tuberculosos de Huipulco y que vio iniciada su actividad en 1936, en las entonces afueras de la Ciudad de México, a las orillas del pueblo de Tlalpan.

Alfonso Reyes inicia su Visión de Anáhuac con un epígrafe que resume lo que pensaba él de la Ciudad de México e invita al recién llegado a reflexionar lo mismo, "Viajero: has llegado a la región más transparente del aire». Si bien Reyes se refiere a toda la zona que corresponde al valle del Anáhuac, es la zona de Tlalpan, a los pies del macizo montañoso donde se erige el volcán del Ajusco, la que cumplía con creces las características climatológicas consideradas ideales para ofrecer a enfermos tuberculosos una mínima posibilidad de curación. Su estabilidad en la temperatura y la humedad del ambiente, el flujo adecuado de corrientes de aire, su acceso a fuentes primarias de nutrientes, entre otros factores, hicieron que los terrenos de Huipulco fueran escogidos por encima de las otras dos localizaciones contempladas, una en la zona de Las Cruces, Cuajimalpa, y la otra en el área de Río Frío al lado de la princesa durmiente del Iztaccíhuatl (estas 
localizaciones con problemas de comunicación que balancearon la decisión en favor de Tlalpan).

El Dr. Donato G. Alarcón describe cómo su interés por la tuberculosis lo llevó a encargarse y ser el primer director del entonces Sanatorio: «En los primeros días de febrero de 1935 fui llamado por el Dr. Leónides Guadarrama Oficial Mayor del Departamento de Salubridad, para tener una entrevista con el Dr. Abraham Ayala González recién nombrado Jefe de ese Departamento, quien me manifestó durante la primera conversación que un problema que deseaba atender inmediatamente era la terminación del Sanatorio para Tuberculosos ubicado en Huipulco, cuya construcción se había iniciado seis años antes y que tenía suspendido todo trabajo constructivo desde hacía más de tres años».2

Describe el Dr. Alarcón haber encontrado el edificio de administración y tres pabellones «...con gran amplitud, tan grande que eso permitió aumentar el cupo desde el inicial que sólo era de 180 camas en los tres pabellones...» Y reconocía la problemática de su distribución horizontal por estar «...unidos a la administración por largos pasillos, por lo que se hacía un recorrido muy grande para observar todo, pues cada pabellón tenía 80 metros de largo». Él no participó de su diseño ni de su construcción y, a pesar de no contar con áreas para el tratamiento «moderno» de la tuberculosis (que en esa época favorecía las distintas metodologías de producir colapso de las cavernas), reconoció que se trataba de una magna obra para beneficio de los pacientes del país: «Acompañado por el Dr. Aquilino Villanueva hice una visita a la construcción suspendida por más de tres años. Había sido planeada por una comisión de la que formaron parte el mismo Dr. Aquilino Villanueva y el Dr. Manuel Gea González, pero no tuve precisiones a ese respecto. Pude ver los planos de la institución que se proyectó siguiendo lineamientos de los sanatorios europeos, con ideas que por los años transcurridos no eran vigentes, pero la obra en general, por lo que ya estaba construido, era una grandiosa realización para el país y quizás para la América Latina».

Si bien el Sanatorio de Huipulco fue basado en los conceptos constructivos de los sanatorios europeos y norteamericanos construidos entre bosques y montañas, distaba mucho de ser igual que ellos. En La Montaña Mágica, Thomas Mann describió sus experiencias al visitar algunos de ellos en el área de Davos, Suiza. ${ }^{3}$ El mítico Sanatorio Internacional Berghof está basado en instituciones existentes en época de Mann y que aún persisten como el Waldsanatorium y, sobre todo, el Schatzalpsanatorium, ahora convertidos en lujosos hoteles frecuentados por aquellos allegados al poder político y económico mundial. ${ }^{4}$
Ya entonces dichos sanatorios eran exclusivos para personas pudientes, muy distinto al recién inaugurado Sanatorio de Huipulco, el cual siempre ha tenido una vocación de solidaridad social para con los que menos oportunidad económica tienen. Y sin embargo, podemos asegurar que desde entonces el Sanatorio de Huipulco, y el ahora INER, sí han constituido una verdadera y mágica montaña para los miles de pacientes que solicitan ser atendidos en sus instalaciones (de 6 niveles socioeconómicos existentes en la actualidad, de manera histórica, el $90 \%$ corresponde a los tres más bajos donde el apoyo que se les brinda va del 85 al 95\% del costo que representa su atención).

Si bien el INER ha sido un hospital solidario socialmente con los necesitados, siempre ha mantenido la dignidad de sus instalaciones, incrementando con el paso de los años su capacidad tecnológica allegándose de equipamientos modernos necesarios en cualquier hospital de clase mundial encargado de manejar pacientes respiratorios (baste mencionar ejemplos como el tomógrafo computarizado multicorte recién instalado gracias a la Administración del Patrimonio de la Beneficencia Pública de nuestro país, equipos de ventilación mecánica avanzada, sistema de oxigenación de membrana extracorpórea, ultrasonido endobronquial, láser y crioterapia endobronquial, así como equipos de diagnóstico molecular Gene-Xpert de infecciones como $\mathrm{TB}, \mathrm{VIH}$ y otras). Además, la calidez del trato ofrecido por todos sus trabajadores siempre ha sido reconocida.

A pesar de su carácter público, con un inicio en el manejo exclusivo de la tuberculosis y siendo los de menor capacidad económica los más frecuentemente afectados y atendidos, el INER/Sanatorio de Huipulco nunca ha sido objeto de descripciones demeritorias. George Orwell describió su experiencia como paciente tuberculoso en el Hôpital Cochin de París en un conocido ensayo titulado Cómo Mueren los Pobres. Encontrándose en París en marzo de 1929, contrajo «la grippe» y fue internado para su tratamiento; su situación económica no era buena por lo que quedó en esa institución pública. La descripción que hace dista mucho de lo que uno esperaría de la Europa del siglo XX; en los últimos párrafos de su escrito reflexiona sobre su experiencia y sobre la manera en que la muerte se torna una cuestión cotidiana, casi banal, en los grandes hospitales: «...la gran cosa es morir en tu propia cama y mejor aún morir en tus propias botas. No importa la amabilidad ni la eficiencia, en cada hospital la muerte será un cruel detalle escuálido, algo tal vez muy pequeño para ser mencionado pero que deja terribles memorias que surgen de la prisa, el apiñamiento, la impersonalidad de un lugar donde cada día personas mueren entre extraños... El pavor a los 
hospitales probablemente aún sobrevive entre los muy pobres, $y$ en todos nosotros apenas recientemente ha desaparecido. Es un parche oscuro no muy por debajo de la superficie de nuestras mentes. He dicho con anterioridad que cuando fui internado en el pabellón del Hôpital $X$ tenía la consciencia de una extraña sensación de familiaridad. Lo que la escena me recordaba, claro, eran los apestosos hospitales llenos de dolor del siglo diecinueve, los cuales nunca había conocido pero de los que mantenía un conocimiento tradicional. $Y$ algo, tal vez el doctor vestido de negro con su maletín oscuro, o tal vez solo el olor enfermizo, hicieron el extraño truco de desenterrar de mi memoria ese poema de Tennyson, El Hospital de Niños, el cual no había yo recordado en más de veinte años...».,5

Quién va a culpar a Orwell por su experiencia en el hospital y como portador de una enfermedad mortal sin cura en ese entonces (murió en 1950 de tuberculosis ya sin poder responder a los incipientes tratamientos quimioterápicos que comenzaban a aparecer). Sin duda los hospitales no son lugares agradables, pero el Sanatorio de Huipulco, y ahora el INER, han hecho su máximo esfuerzo para ofrecer a sus pacientes un área digna, agradable, útil para ellos.

Otro enfermo tuberculoso que vivió épocas más felices desde el punto de vista terapéutico y aún así expone sus experiencias con la enfermedad en toda su obra es Camilo José Cela. En Pabellón de Reposo describe magistralmente varios aspectos del padecer fímico pero, sobre todo, cómo la enfermedad iguala al ser humano en su esencia más profunda, en su humanidad: «...los tuberculosos han dejado de ser abogados, de ser ingenieros, comerciantes, pintores, novios, insatisfechos amantes; han dejado en un sitio ya remoto la carga pesadísima de sus jamás iguales caracteres... ahora ya no son más que enfermos, que enfermos del pecho».7 Por algo dicen que la enfermedad es el más cruel de los mecanismos de igualación social; claro está que en algunas sociedades esa igualación nunca ha sido completa y, parafraseando a Orwell, siempre existen unos más iguales que otros. El INER/Sanatorio de Huipulco nunca ha sido ni será un hospital lujoso y, sin embargo, es capaz de tratar igual a sus pacientes independientemente del rango social al que pertenezcan.

Desde su inicio el Sanatorio de Huipulco era digno y daba servicio a pacientes que llegaban a pasar buenas temporadas en él en tanto intentaban mejorar su salud (la idea de curación habría de esperar la llegada de los medicamentos antituberculosos). De acuerdo con los lineamientos establecidos por el mismo Dr. Alarcón, se daba preferencia a aquellos pacientes que se consideraban candidatos adecuados para ser sometidos a lo que entonces se conocía como tratamiento activo de la tuberculosis. Pronto se llenaron las 180 camas disponibles con enfermos sometidos a neumotórax, plombajes, sección de adherencias pleurales, frenicectomías y toracoplastías que en ese entonces constituían la moda terapéutica prevaleciente internacional.

Seguro que muchos de sus pacientes presentaron dudas existenciales sobre el sometimiento a uno u otro tipo de tratamiento para los problemas que sufrían. De nuevo Camilo José Cela lo describe acuciosamente: « ¿Para qué ha servido esta plastía, que me ha deformado el cuerpo y va en camino de torcerme el espíritu? ¡Ah, si yo hubiera tenido a quien preguntar, ¿qué hago?, ¿me opero?, ¿no me opero?; si yo hubiera tenido a quien pedir un poco de cariño, un poco nada más del mucho cariño que necesito!».

La vocación del INER siempre ha incluido dosis altas de aquello que Cela pedía; por la vocación humana y humanista de sus trabajadores, pero también por vocación profesional al insertar programas recientes de apoyo a pacientes y familiares en situaciones difíciles, así como profundizar en programas de manejo paliativo.

La primer plantilla reunida por el Dr. Alarcón podría haber tomado su lugar en cualquier Schatzalpsanatorium del mundo y él lo describe mejor: «El pabellón No. 1 para hombres se encomendó al Dr. Ismael Cosío Villegas, el No. 2 también para hombres al Dr. Aniceto del Río y me hice cargo del No. 3 para mujeres. La planta inicial de médicos incluía a los Dres. Miguel Jiménez, Dr. Mario Vergara Soto, Dr. Fernando Rébora, Dr. Manuel Alonso, Dr. Horacio Rubio Palacios, Dr. Xavier Hernández, como clínicos, el Dr. Alfredo Iglesias se hizo cargo del laboratorio, y el Dr. Alejandro Celis de roentgenología, Dr. Ricardo Tapia Acuña otorrinolaringólogo, Dr. Isidoro Sota García dentista y Prof. Arturo Madrid de la farmacia» (sic). Este fue el grupo inicial del que habría de iniciar la evolución de un sanatorio dedicado a una enfermedad para terminar en lo que actualmente es, ochenta años después: toda una Institución dedicada a las enfermedades del aparato respiratorio.

La transformación que ha hecho del Sanatorio de Huipulco convertirse en Instituto Nacional de Enfermedades Respiratorias responde por un lado a la evolución de las especialidades dedicadas al aparato respiratorio y, por el otro, a lo que resulta casi un cliché de la múltiplemente mentada transición epidemiológica en la que se encuentra nuestro país. Enfermedades infecciosas coexistiendo con enfermedades crónico degenerativas con un impacto importante en lo que se conoce como la Carga de Morbimortalidad de nuestra sociedad.

En efecto, dicha carga de enfermedad (bajo el concepto de GBD o Global Disease Burden), muestra 
claro cómo en nuestro país las enfermedades respiratorias ocupan un lugar predominante como causa de discapacidad y pérdida de años de vida ajustados a enfermedad (los famosos DALY's utilizados hoy como parámetro de medición), según datos para el año 2013 producidos por el consorcio manejado por el Instituto de Métrica y Evaluación (de la Universidad Washington, en conjunto con la Organización Mundial de la Salud, la Escuela de Salud Pública de Harvard, la Escuela de Salud Poblacional de la Universidad de Auckland y el Banco Mundial, auspiciados por la Fundación Bill y Melinda Gates; http://www.healthdata.org/ gbd). Las infecciones respiratorias agudas (neumonías e influenza), la tuberculosis asociada o no a infección por virus de la inmunodeficiencia humana, coexisten en nuestro país como causa del padecer de su sociedad con la enfermedad pulmonar obstructiva crónica, el cáncer pulmonar, el asma, las enfermedades pulmonares intersticiales y otras menos frecuentes. Las enfermedades respiratorias son una carga importante en el padecer de los seres humanos que conforman nuestra sociedad. Como bien menciona la revista Lancet en un recién editorial, el pulmón es un órgano magnífico pero requiere atención a lo largo de toda la vida. ${ }^{8}$ Alguien tiene que hacerlo; el INER asume su responsabilidad.

Dentro de las primeras causas de enfermedad en nuestro país, las del aparato respiratorio ocupan el $62 \%$ (infecciones respiratorias agudas, otitis, asma, neumonía y faringitis). ${ }^{9}$ Por su lado, las enfermedades respiratorias ocupan cuatro de las primeras veinte causas de mortalidad (EPOC, bronquitis -sic, enfisema -sic, asma, influenza/neumonías y cáncer broncogénico)..$^{10}$ Atender estas enfermedades de manera racional y bajo evidencia científica constituye una obligación moral primordial del INER. Pero no sólo eso, sino también constituyen nuestro deber ético; por un lado, el hacer nuestro máximo esfuerzo por participar en la producción mundial de ese conocimiento científico y, por otro lado, comunicar ese conocimiento a médicos en preparación en las especialidades respiratorias, a enfermeras, químicos, terapeutas, psicólogos y otros conformantes del equipo de atención médica pero, sobre todo, a los pacientes y sus familiares que atendemos cotidianamente. Sin esa enseñanza del conocimiento, que científicamente sometemos a prueba y validación de manera cotidiana, poco lograremos para que la sociedad comprenda que el trabajo que realizamos es el mejor camino para intentar modificar esa "carga de enfermedad» con la que tiene que lidiar y, finalmente, acepte nuestro trabajo como algo bueno, como aliados suyos al enfrentar sus vicisitudes de salud.
En su devenir, el Sanatorio de Tuberculosos de Huipulco se transformó en Instituto Nacional de Enfermedades Respiratorias. Ochenta años han pasado y seguimos lidiando con la tuberculosis más otras enfermedades que conforman una carga pesada de padecimiento a la sociedad.

En su gran obra titulada Los Viajes Hacia Varias Naciones Remotas del Mundo en Cuatro Partes por Lemuel Gulliver Primero Cirujano y Luego Capitán de Varios Navíos, su autor, Jonathan Swift, hace una crítica a la sociedad británica de la primera mitad del siglo XVIII. En uno de los capítulos describe la nación de Luggnagg donde Gulliver encuentra a los «struldbrugs», seres cuya inmortalidad les previene de sucumbir a enfermedades que afectan a otros seres. Sin embargo eso, paradójicamente, se vuelve una fuente infinita de padecer ya que su inmortalidad no conlleva la eterna juventud; al contrario, los struldbrugs envejecen de tal manera que al cumplir ochenta años mejor son declarados oficialmente muertos, aislados de la sociedad y pasan el tiempo enfermando sin morir, vagando por el país con la mirada perdida y sin ninguna ilusión que les estimule. ${ }^{11}$

Tal vez el mensaje que nos deja Swift sea no pensar que nuestro objetivo médico deba lograr la ausencia total de enfermedad y la posibilidad de una inmortalidad, sino más bien pensar en que la longevidad que logre el ser humano debe acompañarse de una calidad de vida que permita poder disfrutar de ella plenamente. Como institución debemos reflexionar sobre lo mismo; tal vez ya no estemos en la región más transparente del aire pero, al volvernos octogenarios, sí podemos pensar cómo evitar caer en la pasividad deprimente de los struldbrugs y alejarnos del riesgo de que nos declaren institucionalmente muertos; podemos evitar el pesimismo desesperante del Dr. Efimich en el relato de Chéjov.

Pienso que el INER, en su evolución, da muestras fehacientes en contra de esto. Su vitalidad, su creatividad intelectual, su ánimo humanista y su interés en servir a la sociedad se muestra diario. Aquellos que nos antecedieron dentro de la Dirección Médica y la Subdirección de Atención Médica, sin duda han contribuido a hacernos participar de la sensación newtoniana atribuida a Bernardo de Chartres de poder ver más allá por estar parados sobre los hombros de los que nos antecedieron.

El INER, a sus ochenta años, se encuentra vivo y se ha convertido en el Aleph del conocimiento y la atención respiratoria de nuestro país, precisamente como dice el epígrafe de este escrito: el lugar donde están, sin confundirse, todos los lugares del orbe, vistos desde todos los ángulos. ¡Enhorabuena y larga vida! 


\section{REFERENCIAS}

1. Sontag S. Illness as metaphor. Farrar, Straus and Giroux: New York; 1977.

2. Alarcón GD. Los primeros doce años de actividad del Sanatorio para Tuberculosos de Huipulco. En el cincuentenario de su inauguración. Neumol Cir Torax 2010;69(4):218-228.

3 Mann T. The magic mountain. Woods JE (traductor). Vintage International: New York; 1995.

4. McGrane S. To the magic mountain! Febrero 17, 2014. The New Yorker

5. Davies P. How the poor die. BMJ 2012;345:e6699. doi: 10.1136/bmj.e6699. (Published 4 October 2012).

6. Orwell G. How the poor die. Access date: 2016 July 25. Available from: http://www.orwell.ru/library/articles/ Poor_Die/english/e_pdie

7. Cela CJ. Pabellón de reposo. Madrid, España: Destino; 1979.

8. The lung: a magnificent organ that needs lifelong attention (Editorial). Lancet 2016;387(10030):1789. doi: 10.1016/S0140-6736(16)30357-9.
9. Access date: 2016 June 9. Available from: http://www. epidemiologia.salud.gob.mx/anuario/2014/morbilidad/ nacional/veinte_principales_causas_enfermedad_nacional_grupo_edad.pdf

10. Access date: 2016 June 9. Available from: http:// www3.inegi.org.mx/sistemas/sisept/Default. asp $? \mathrm{t}=$ mdemo107\&s $=$ est $\& \mathrm{c}=23587$

11. Swift J. Gulliver's travels into remote nations of the world. Access date: 2016 July 25. Available from: http:// www.literatureproject.com/gulliver-travel/

\section{$\triangle$ Correspondencia:}

Dr. Patricio Santillán Doherty, Editor en Jefe de NCT, Director Médico, Instituto Nacional de Enfermedades Respiratorias Ismael Cosío Villegas, Ciudad de México. Calzada de Tlalpan 4502, colonia Sección XVI, Código postal 14080,

Del. Tlalpan, Ciudad de México.

Correo electrónico: patricio.santilland@gmail.com

Los autores declaran no tener conflicto de intereses. 